Mitteilungen der Österreichischen Geographischen Gesellschaft,

151. Jg. (Jahresband), Wien 2009, S. 141-157

\title{
Klimä̈nderung: Mögliche Auswirkungen des Temperaturanstiegs auf den Weinbau in Der Wachau
}

\author{
Alexander WIMMER, Wien* \\ mit 7 Abb. und 5 Tab. im Text
}

\section{INHALT}

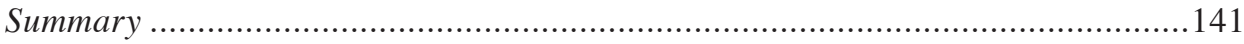

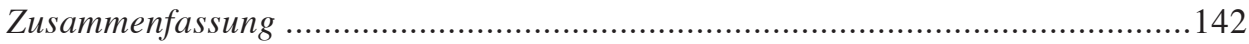

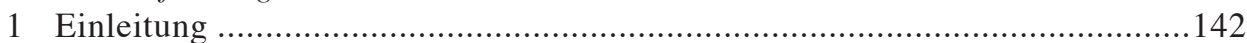

2 Österreichs bekanntestes Weinbaugebiet: die Wachau .................................142

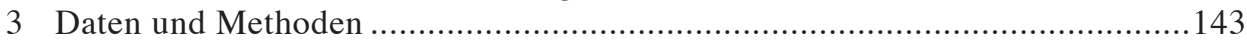

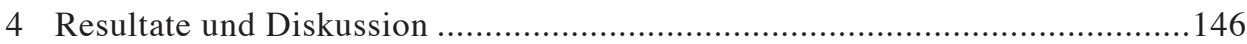

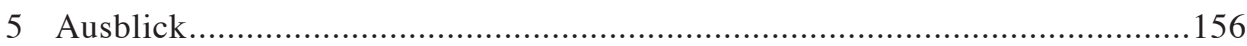

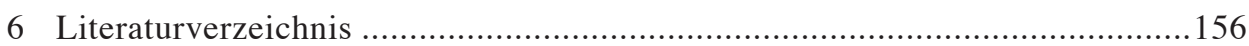

\section{Summary}

Climate change: Potential effects of temperature increase on viticulture in the Wachau region

In the article the possible changes in viticulture brought about by increasing temperatures caused by climate change in the Wachau region (Lower Austria) are investigated. For an analysis of the past temperatures in the Wachau, weather data from 1971-2006 are processed and evaluated using statistical procedures. The results of a climate scenario developed for Austria is used for assessment of future temperature increases. The investigation and illustration of the influence of temperature increase on viticulture is carried out by using the heat summation model of GLADSTONES and by comparing the Wachau region with the region of Bordeaux (France).

\footnotetext{
* Mag. Dr. Alexander WIMmER, MBA, Langackergasse 13/2, A-1190 Wien; e-mail: alexander_wimmer@ ymail.com
} 


\section{Zusammenfassung}

In diesem Beitrag werden die durch steigende Temperaturen möglichen Veränderungen im Weinbau in der Region Wachau untersucht. Für die Analyse der Temperaturen in der Vergangenheit werden Wetterdaten von 1971-2007 mit statistischen Verfahren aufbereitet und ausgewertet. Für die Einschätzung der zukünftigen Temperaturen werden die Resultate eines für Österreich entwickelten Klimaszenarios verwendet. Die Untersuchung und Darstellung des Einflusses der Temperatur auf den Weinbau wird mit dem Wärmesummenmodell von GLADSTONES und einem Vergleich der Weinbauregion Wachau mit der Region um Bordeaux (Frankreich) durchgeführt.

\section{Einleitung}

Es gibt eine Vielzahl von unterschiedlichen Klima- und Wetterfaktoren, die Einfluss auf das Wachstum der Rebe und die Weinqualität haben. Die Länge der Wachstumsperiode und die Temperatur sind kritisch, da diese Faktoren im Wesentlichen bestimmen, ob bestimmte Rebsorten zur Reife gebracht werden können. Trauben brauchen Wärme, um reif zu werden. Aber um feine Weine zu erzeugen, sind „kühle“ Temperaturen (PrIEwe 1998, S. 18) notwendig. Dadurch wird die Zuckerentwicklung in den Trauben gebremst und verhindert, dass die Weine zu alkoholisch und schwer werden. Durch die Klimaänderung und den damit verbundenen Temperaturanstieg ergeben sich neue Herausforderungen für den Weinbau. Der vorliegende Beitrag behandelt folgende Themenstellungen:

1. In welchem Ausmaß hat ein Temperaturanstieg in der Wachau bereits stattgefunden?

2. Welche Temperaturverhältnisse sind in der Wachau in den nächsten Jahrzehnten zu erwarten?

3. Welche möglichen Auswirkungen hat der Temperaturanstieg auf den Weinbau in der Wachau?

\section{2 Österreichs bekanntestes Weinbaugebiet: die Wachau}

Die Wachau ist eine etwas über $30 \mathrm{~km}$ lange Flusslandschaft zwischen Krems an der Donau und Melk. Die Wachau ist klimatisch sehr komplex, da hier aus dem Osten in das Tal hereinreichende pannonische Einflüsse mit westlich-kontinentalen Strömungen zusammenstoßen. Die Durchschnittstemperatur während der Vegetationsperiode von April bis Oktober für Krems an der Donau ${ }^{1)}$ betrug im Zeitraum 1971-2000 14,7 ${ }^{\circ} \mathrm{C}$, damit entspricht die Wachau einem „kühlen Weinbaugebiet“ (vgl. JonEs 2006, S. 24). Die Rebkulturen ziehen sich auf den Terrassen bis in ca. $450 \mathrm{~m}$ Seehöhe hinauf, also 250 m über dem Donaustrom. Auf einer Rebfläche von 1.373 Hektar wachsen vor- 
wiegend die zwei Hauptsorten Grüner Veltliner (710 ha) und Riesling (185 ha) (vgl. ÖWM 2006). Das Profil der Weinbauregion Wachau ist gänzlich auf diesen zwei Leitsorten aufgebaut.

\section{Daten und Methoden}

Für die Analyse der Temperatur der vergangenen Jahrzehnte in der Wachau wurden umfangreiche Daten der Wetterstationen Hohe Warte und Krems an der Donau der Zentralanstalt für Meteorologie und Geodynamik ausgewertet (ZAMG 2007 \& 2008a). Es wurde eine Regressionsanalyse durchgeführt, um die Ausprägungen der abhängigen Variablen (Temperatur, Wärmesummenindex) aufgrund der Ausprägungen der unabhängigen Variablen (Jahr) zu bestimmen. Es wurde somit eine einseitige Abhängigkeit unterstellt. Zur Überprüfung der Signifikanz des linearen Regressionsmodells wurde der „F-Test“ durchgeführt, für die Signifikanz der Steigung der Regressionsgeraden der ,t-Test“.

Für die Einschätzung der zukünftigen Temperatur in der Wachau wurden die Resultate des Projektes reclip:more (Research for Climate Protection:Model Run Evaluation) verwendet. reclip:more stellt kleinräumige Daten für die regionale Klimafolgenforschung zur Verfügung. Die Klimaszenarien wurden in mesoskaliger Auflösung ( 10 km) mittels zweier regionaler Klimamodelle (RCM) und nachgeschaltetem Downscaling mit Zielauflösung von $1 \mathrm{~km}$ generiert (reclip:more 2007a).

Die phänologischen ${ }^{2)}$ Daten für die Rebblüte des Grünen Veltliners am Standort Sandgrube in Krems an der Donau für den Zeitraum 1965-2007 wurden von der Landwirtschaftlichen Fachschule Krems an der Donau zur Verfügung gestellt. Die Rebblüte wird aufgrund visueller Beobachtung festgestellt. Bis zu 90\% der Variabilität der Eintrittstermine der phänologischen Phasen von Jahr zu Jahr können durch die Variabilität der bodennahen Lufttemperatur erklärt werden (Koch \& SCHEIFINGER 2004, S. 18).

Als Kriterium zur Beschreibung der regionalen Verhältnisse für die physiologische Entwicklung des Rebstocks wurde der Wärmesummenindex nach GLADSTONES (1992) $\mathrm{WI}_{\mathrm{G}}$ verwendet.

Die Wärmesumme ist die Summe bestimmter Temperaturwerte innerhalb eines definierten Zeitraumes. Die physiologische Entwicklungszeit ergibt sich aus der Wärmesumme, die eine Pflanze benötigt, um ihre Entwicklung abzuschließen. Folgende Parameter wurden verwendet, um den Temperatureffekt auf das Wachstum und die Entwicklung abzubilden (vgl. Boulton et al. 1998, S. 30):

\footnotetext{
1) Krems an der Donau ist die nächstgelegene Wetterstation der Zentralanstalt für Meteorologie und Geodynamik (ZAMG) zum Weinbaugebiet Wachau. In den weiteren Ausführungen werden daher die Daten der Wetterstation Krems an der Donau der Analyse der Temperaturverhältnisse in der Weinbauregion Wachau zugrunde gelegt. Krems an der Donau selbst liegt nicht in der Wachau.

2) Phänologie ist die Wissenschaft von den jahreszeitlich bedingten Erscheinungsformen und dem Entwicklungszyklus bei Tieren und Pflanzen (Косн \& Scheifinger 2004, S. 17). Beim Rebstock sind dies in zeitlicher Reihenfolge (Boulton et al. 1998, S. 27ff.): Austrieb, Blüte, Fruchtansatz, Véraison (Reifebeginn der Trauben), Maturation (Reife), Weinlese und als Abschluss der Blattfall (Laubfall).
} 
- unterer Temperatur-Schwellenwert: die Entwicklung der Pflanze setzt ein bzw. stoppt, wenn die Temperatur unter den Schwellenwert fällt;

- oberer Temperatur-Schwellenwert: die Entwicklungsrate der Pflanze flacht bei Überschreiten des Schwellenwertes ab.

Die Wärmesumme, welche eine Pflanze benötigt, um ihre Entwicklung abzuschließen, ist immer gleich. Das heißt, dass mit dem Parameter Temperatur bestimmt werden kann, ob der Wärmebedarf einer bestimmten Pflanze (Rebe) unter den vorherrschenden Verhältnissen erreicht wird. Die Wärmesumme eines Tages, eines sogenannten Growing Degree Day (GDD), berechnet sich folgendermaßen:

$$
G D D=\left(t_{\max }+t_{\min }\right) / 2-t_{\text {base }}
$$

GDD $=$ Growing Degree Day (Wärmegradtag)

$\mathrm{t}_{\max }=$ tägliche Maximumtemperatur

$\mathrm{t}_{\min }=$ tägliche Minimumtemperatur

$\left(\mathrm{t}_{\max }+\mathrm{t}_{\min }\right) / 2$ entspricht der Durchschnittstemperatur $\mathrm{t}$

$\mathrm{t}_{\text {base }}=$ Basistemperatur (als Tagesmittel zu verstehen), ab der das Wachstum der Rebe einsetzt $=$ unterer Temperatur-Schwellenwert

$\mathrm{t}_{\text {base }}$ ist für die verschiedenen Pflanzen unterschiedlich und wird experimentell ermittelt. Für die Rebe wird t ${ }_{\text {base }}$ generell mit $10^{\circ} \mathrm{C}$ angenommen, d.h., ab dieser Temperatur setzt das Wachstum der Rebe ein, während bei niedrigeren Temperaturen das Wachstum eingestellt ist.

Zum Beispiel würde über eine 5-Tages-Periode mit einem Maximum von $30^{\circ} \mathrm{C}$ und einem Minimum von $15^{\circ} \mathrm{C}$ jeder Tag zur Wärmesumme $\left.[(30+15) \div 2)\right]-10$ $=12,5$ GDD beitragen und die Wärmesumme $5 \times 12,5$ GDD $=62,5$ GDD betragen.

\section{Wärmesummen-Index nach GLADSTONES - Berechnung}

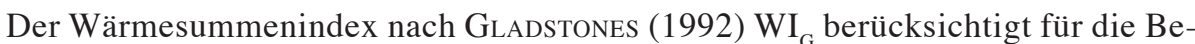
rechnung der Wärmesumme nur den Bereich der Durchschnittstemperatur $t$ zwischen $10^{\circ} \mathrm{C}$ und $19^{\circ} \mathrm{C}$ (biologisch effektiver ,Temperaturkorridor" ${ }^{\prime \prime}$. Das ist jener Bereich, in dem die Rebe das größte physiologische Wachstum aufweist. Unter $10^{\circ} \mathrm{C}$ wird das Wachstum eingestellt, über $19^{\circ} \mathrm{C}$ flacht die Wachstumskurve sehr stark ab und das physiologische Wachstum geht gegen Null. Die Berechnung des $\mathrm{WI}_{\mathrm{G}}$ erfolgt von April bis Oktober, da in der Wachau die Trauben für die Smaragdweine ${ }^{3)}$ erst im Oktober, manchmal sogar erst im November, gelesen werden.

Einige Rebsorten reifen früh und können erfolgreich in kühlem Klima mit kurzen Vegetationsperioden angebaut werden; andere Traubensorten reifen spät und benötigen eine lange Vegetationsperiode mit einer hohen biologisch effektiven Wärmesumme (Bereich zwischen $10^{\circ} \mathrm{C}<\mathrm{t} 19^{\circ} \mathrm{C}$, in dem das größte physiologische Wachstum der Rebe stattfindet), um zur vollen Reife zu gelangen. GLADSTONES unterteilt die Traubensorten in acht Reifegruppen, mit den dazugehörigen Anforderungen an die biologisch effektive Wärmesumme.

\footnotetext{
3) Smaragd ist die Bezeichnung für die besten und wertvollsten Weine der Wachau ab einem Alkoholgehalt von $12,5 \%$ Vol.
} 


$$
\begin{aligned}
& W I_{G}=\sum_{1=01.04}^{i=31.10} K_{G} * G D D_{i} \\
& t \leq 10^{\circ} \Rightarrow G D D=0 \\
& 10^{\circ}<t<19^{\circ} \Rightarrow G D D=t-10^{\circ} \mathrm{C} \\
& t \geq 19^{\circ} \Rightarrow G D D=9
\end{aligned}
$$

$\mathrm{K}_{\mathrm{G}}=$ Koeffizient (Gladstones 1992): Faktoren zur Anpassung der wachstumseffektiven Temperatur $\left(\mathrm{t} \geq 10^{\circ} \mathrm{C}\right)$ an die Tageslänge, abhängig vom Monat und der geographischen Breite.

\begin{tabular}{|c|c|c|}
\hline $\begin{array}{l}\text { GDD- } \\
\text { Summe }\end{array}$ & Rote Weine & Weiße oder Rosé-Weine \\
\hline $\begin{array}{l}\text { Gruppe } 1 \\
1050 \mathrm{Tag}^{\circ}\end{array}$ & & Madeleine, Madeleine-Sylvaner \\
\hline $\begin{array}{l}\text { Gruppe } 2 \\
1100 \mathrm{Tag}^{\circ}\end{array}$ & Blauer Portugieser, St. Laurent & $\begin{array}{l}\text { Chasselas, Müller-Thurgau, Siegerrebe, } \\
\text { Bacchus, Pinot Gris, Muscat Ottonel, Roter } \\
\text { Veltliner, Pinot Noir, Meunier }\end{array}$ \\
\hline $\begin{array}{l}\text { Gruppe } 3 \\
1150 \mathrm{Tag}^{\circ}\end{array}$ & $\begin{array}{l}\text { Pinot Noir, Meunier, Gamay, Dolcet- } \\
\text { to, Bastardo, Tinta Carvalha, Tinta } \\
\text { Amarella }\end{array}$ & $\begin{array}{l}\text { Traminer, Sylvaner, Scheurebe, Elbling, } \\
\text { Morio-Muskat, Kerner, Grüner Veltliner, } \\
\text { Chardonnay, Aligoté, Melon, Sauvignon } \\
\text { Blanc, Frontignac, Pedro Ximenes, Verdelho }\end{array}$ \\
\hline $\begin{array}{l}\text { Gruppe } 4 \\
1200 \mathrm{Tag}^{\circ}\end{array}$ & $\begin{array}{l}\text { Malbec, Durif, Zinfandel, Schiava, } \\
\text { Tempranillo, Tinta Madeira, Pinotage, } \\
\text { Zweigelt, Blauburger }\end{array}$ & $\begin{array}{l}\text { Sémillon, Muscadelle, Riesling, Welschries- } \\
\text { ling, Furmint, Leanyka, Harslevelu, Sercial, } \\
\text { Malvasia Bianca, Cabernet Franc }\end{array}$ \\
\hline $\begin{array}{l}\text { Gruppe } 5 \\
1250 \mathrm{Tag}^{\circ}\end{array}$ & $\begin{array}{l}\text { Merlot, Cabernet Franc, Shiraz, } \\
\text { Cinsaut, Barbera, Sangiovese, } \\
\text { Touriga, Blaufränkisch }\end{array}$ & $\begin{array}{l}\text { Chennin Blanc, Folle Blanche, Crouchen, } \\
\text { Roussane, Marsanne, Viognier, Taminga, } \\
\text { Cabernet Sauvignon }\end{array}$ \\
\hline $\begin{array}{l}\text { Gruppe } 6 \\
1300 \mathrm{Tag}^{\circ}\end{array}$ & $\begin{array}{l}\text { Cabernet Sauvignon, Ruby Cabernet, } \\
\text { Mondeuse, Tannat, Kadarka, Corvina, } \\
\text { Nebbiolo, Ramisco, Alvarelhao, } \\
\text { Mourisco Tinto, Valdiguie }\end{array}$ & $\begin{array}{l}\text { Colomard, Palomino, Dona Brance, } \\
\text { Rabigato, Grenache }\end{array}$ \\
\hline $\begin{array}{l}\text { Gruppe } 7 \\
1350 \mathrm{Tag}^{\circ}\end{array}$ & $\begin{array}{l}\text { Aramon, Petit Verdot, Mataro, Carignan, } \\
\text { Grenache, Freisa, Negrara, Grignolino, } \\
\text { Souzai, Graciano, Monastrell }\end{array}$ & Muscat Gordo Blanco, Trebbiano, Montils \\
\hline $\begin{array}{l}\text { Gruppe } 8 \\
1400 \mathrm{Tag}^{\circ}\end{array}$ & Tarrango, Terret Noir & $\begin{array}{l}\text { Clairette, Grenache Blanc, Doradillo, } \\
\text { Biancone }\end{array}$ \\
\hline
\end{tabular}

\begin{tabular}{|l|c|c|c|c|c|c|c|c|}
\hline $\begin{array}{l}\text { Monat / } \\
\text { geogr. Breite }\end{array}$ & Apr & Mai & Jun & Jul & Aug & Sep & Okt & $\begin{array}{l}\text { Durch- } \\
\text { schnitt }\end{array}$ \\
\hline $48^{\circ}$ (Krems) & 1,030 & 1,055 & 1,069 & 1,062 & 1,041 & 1,011 & 0,974 & 1,036 \\
\hline
\end{tabular}

Tab. 1: Faktoren $\mathrm{K}_{\mathrm{G}}$ zur Anpassung der wachstumseffektiven Temperatur (GLADSTONES 1992, S. 63)

Tab. 2: Zuordnung der Rebsorten zu Reifegruppen nach Growing Degree Day (GDD) (Gladstones 1992, S. 67), ergänzt durch den Autor um die Rebsorten Blauburger, Blaufränkisch, St. Laurent, Zweigelt. Die wichtigsten Rebsorten der Wachau (Grüner Veltliner und Riesling) sind hervorgehoben. 


\section{Resultate und Diskussion}

\subsection{Homogenitätsprüfung der Daten der Wetterstation Krems an der Donau}

Da das Klimaregime eines Gebietes mehr oder weniger langzeitliche Schwankungen aufweist, gelten die statistischen Auswertungen der Stationsdaten nur für den Zeitraum, für den die Daten vorliegen (vgl. Olberg et al. 1991, S. 157ff.). Die Daten müssen auch auf Homogenität geprüft werden, da kaum Messreihen existieren, die unter konstanten oder quasikonstanten Bedingungen gewonnen wurden. Viele Faktoren spielen hier eine Rolle, wie die Entwicklung der Mess- und Auswerttechnik (z.B. automatische Aufzeichnung versus Ablesen der Messdaten durch einen Menschen), die Modifikation der Messbedingungen wie Verlegung von Stationen, die Veränderung der Instrumentation oder der Expositionshöhe von Messgeräten, die Modifikation des Messplatzes durch Urbanisierung, Industrialisierung, etc. Dies hat dazu geführt, dass sich die Datensätze aus Teilreihen unterschiedlicher Qualität zusammensetzen (vgl. Olberg et al. 1991, S. 159ff.).

\begin{tabular}{|c|c|c|c|c|c|c|}
\hline \multirow{2}{*}{$\begin{array}{l}\text { Stations- } \\
\text { nummer }\end{array}$} & \multicolumn{2}{|c|}{ vorhandene Daten } & \multicolumn{2}{|c|}{ Geographische } & \multirow{2}{*}{$\begin{array}{l}\text { See- } \\
\text { höhe }\end{array}$} & \multirow{2}{*}{ Ort } \\
\hline & von & bis & Länge & Breite & & \\
\hline 3807 & 01.01 .1948 & 31.05 .1977 & $15^{\circ} 36^{\prime} 00^{\prime \prime}$ & $48^{\circ} 25^{\prime} 00^{\prime \prime}$ & $223 \mathrm{~m}$ & Krems an der Donau \\
\hline 3800 & 01.06 .1977 & 30.09 .1982 & $15^{\circ} 37^{\prime} 00^{\prime \prime}$ & $48^{\circ} 25^{\prime} 00^{\prime \prime}$ & $200 \mathrm{~m}$ & Krems-Landersdorf \\
\hline 3801 & 01.10 .1982 & 31.03 .1996 & $15^{\circ} 37^{\prime} 20^{\prime \prime}$ & $48^{\circ} 25^{\prime} 07^{\prime \prime}$ & $207 \mathrm{~m}$ & Krems an der Donau \\
\hline 3805 & 01.04 .1996 & 30.11 .2007 & $15^{\circ} 37^{\prime} 15^{\prime \prime}$ & $48^{\circ} 25^{\prime} 06^{\prime \prime}$ & $204 \mathrm{~m}$ & Krems an der Donau \\
\hline
\end{tabular}

Tab. 3: Standortdaten der Wetterstationen in Krems an der Donau

Die Homogenitätsprüfung der Stationsdaten von Krems an der Donau erfolgt durch Vergleich der Temperatur-Messdaten mit denen der Station Hohe Warte, 1190 Wien, die zu den Stationen mit besserer Homogenität zählt (vgl. ZAMG 2008b).

Für die Homogenitätsprüfung wird im ersten Schritt die Differenz der Messreihen der Stationen Krems an der Donau und Hohe Warte für die mittlere Tagesminimumtemperatur $\left({ }_{\mathrm{m}} \mathrm{t}_{\min }\right)$ und die mittlere Tagesmaximumtemperatur $\left({ }_{\mathrm{m}} \mathrm{t}_{\max }\right)$ über den Zeitverlauf von 1948 bis 2006 gebildet. Im zweiten Schritt werden die ermittelten Werte in einer Zeitreihe dargestellt, wobei auf der Ordinate die Temperaturdifferenz aufgetragen wird und auf der Abszisse die Jahre. Das wenig erfreuliche Ergebnis und die Bestätigung, dass kaum Messreihen existieren, die homogen sind, zeigt Abbildung 1.

Wird die Datenreihe der Hohen Warte als homogen angesehen, müsste eine konstante Differenz zu Krems an der Donau vorliegen, d.h. die Kurven für ${ }_{m} t_{\text {min }}$ und ${ }_{m} t_{\text {max }}$ müssten parallel zur Abszisse verlaufen, verschoben um die (konstante) Differenz zwischen den Messwerten der Hohen Warte und Krems an der Donau. Die Probleme sind in Abbildung 1 sehr gut ersichtlich. Die Station in Krems an der Donau (Stationsnummern: $3807,3800,3801,3805)$ wurde dreimal verlegt (vgl. Abb. 2) und zeigt 


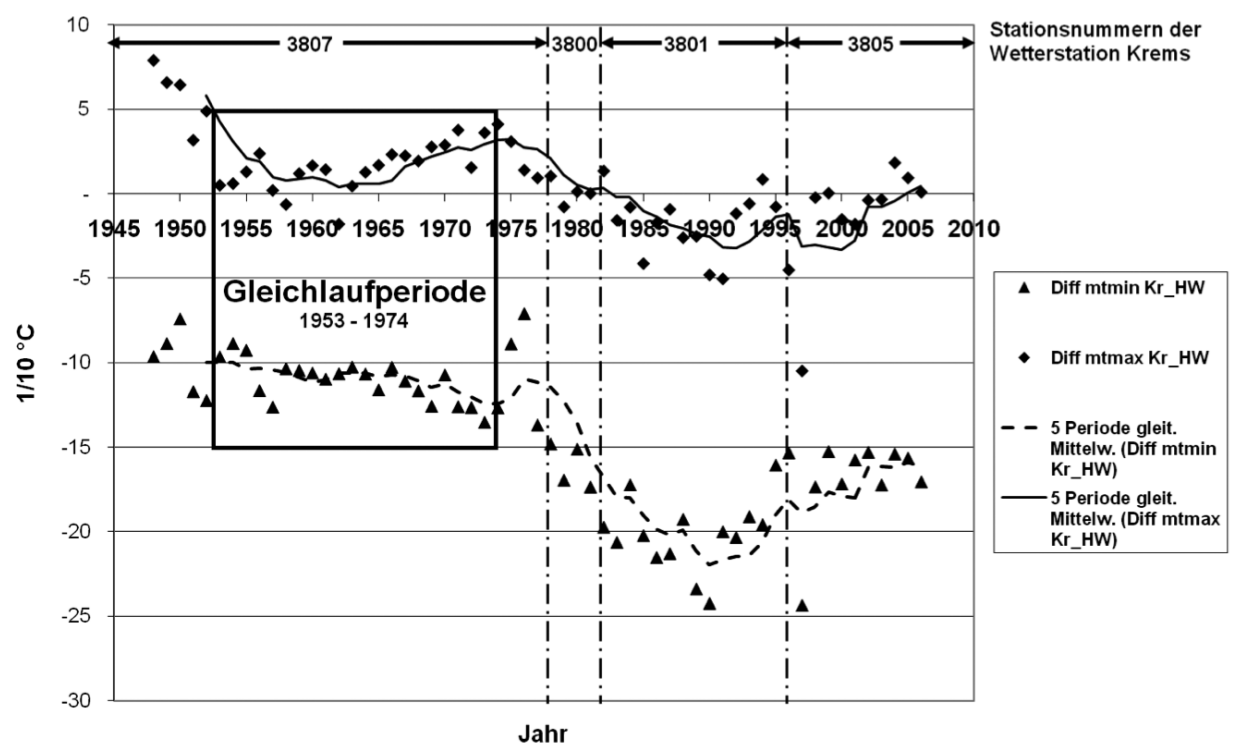

Abb. 1: Verlauf der Temperaturdifferenzen ${ }_{\mathrm{m}} \mathrm{t}_{\min }$ und ${ }_{\mathrm{m}} \mathrm{t}_{\max }$ zwischen Krems an der Donau und Hoher Warte 1948-2006

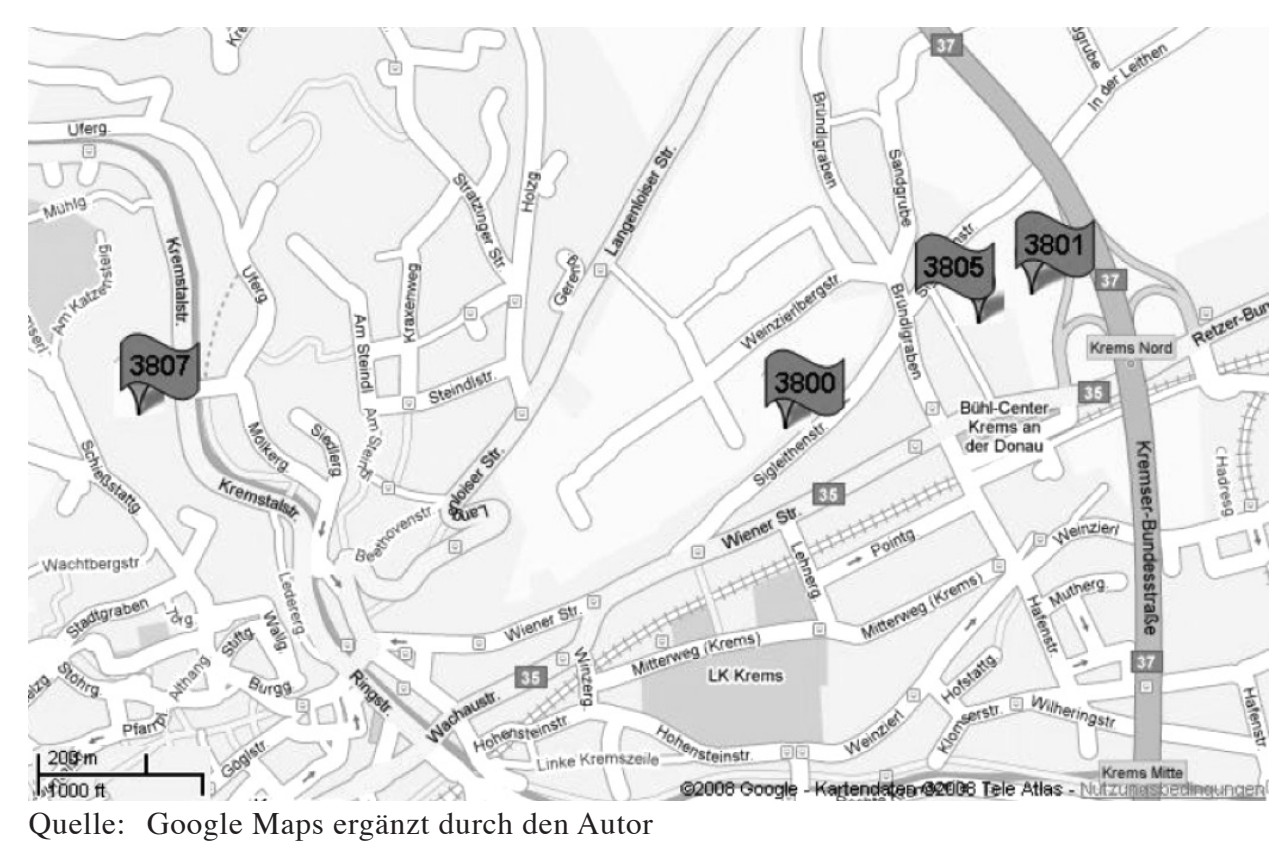

Abb. 2: Standorte der Wetterstationen in Krems an der Donau 
auch sonst Inkonsistenzen, die eine Trendanalyse basierend auf den Messdaten dieser Station nicht zulassen.

Die Stationen 3800 und 3801 erscheinen unter dem Aspekt der Homogenität als besonders problematisch. Hier laufen die Messwerte sprichwörtlich „aus dem Ruder“ und zeigen bei der Minimumtemperatur einen (unerwünschten) Trend von fast $1,5^{\circ} \mathrm{C}$ in 15 Jahren (1977-1992).

Zur Behebung des Homogenitätsproblems wurde eine Korrektur der Daten mittels der „Methode quasikonstanter Differenzen“ durchgeführt (vgl. OlbBerg et al. 1991, S. 162). Diese Methode kann angewendet werden, wenn die Entfernung der Referenzstation (Hohe Warte) zu der zu untersuchenden Station (Krems an der Donau) hinreichend klein ist. In Abbildung 3 sind die Dekorrelationsdistanzen ${ }^{4)}$ für einige Klimaparameter dargestellt. Hier ist ersichtlich, dass für die Temperatur auf Tagesbasis die Dekorrelationsdistanz $533 \mathrm{~km}$ beträgt.

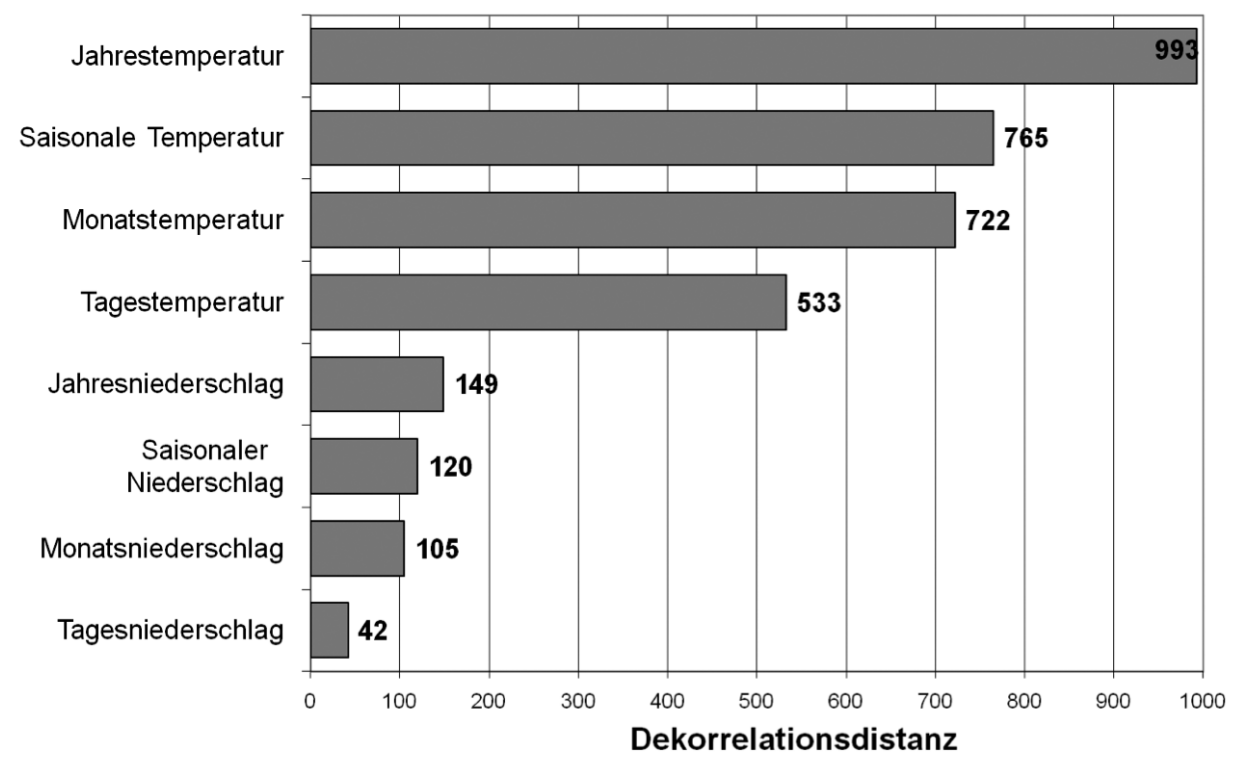

Abb. 3: Darstellung der Dekorrelationsdistanzen $(\mathrm{km})$ mit dem Varianzkriterium $\mathrm{r} 2=0,5$ [SCHEIFINGER, BöHM \& Auer 2003, Grafik vom Autor zur Verfügung gestellt, Tageswerte gültig für Europa, Monats-, Saisonal- und Jahreswerte gültig für das GAR-Gebiet (Greater Alpine Region $\left.4^{\circ}-18^{\circ} \mathrm{E}, 43^{\circ}-49^{\circ} \mathrm{N}\right)$ ]

\footnotetext{
4) Dekorrelationsdistanz: jene Distanz, in der die erklärte Varianz unter 0,5 sinkt. Hierbei unterstellt man, dass die gemeinsame Korrelation linear mit der Entfernung abnimmt. Die Dekorrelationsdistanzen für die jährlichen, saisonalen und monatlichen Zeitreihen wurden aus einem Zeitabschnitt von 100 Jahren (1896-1995) aus dem HISTALP-Datenset, für die täglichen Zeitreihen aus den 4-Jahres-SYNOP-Daten (1999-2002) berechnet.
} 
Zur Korrektur der Daten von Krems an der Donau wurde wie folgt vorgegangen:

1. Aus dem zeitlichen Verlauf der Temperaturdifferenz 1948-2006 wird eine „Gleichlaufperiode" für die Stationen Krems an der Donau und Hohe Warte bestimmt, d.h. eine Periode, in der die Differenzwerte annähernd parallel zur X-Achse verlaufen. Als Gleichlaufperiode wurde der Zeitraum von 1953-1974 gewählt (vgl. Abb. 1). Der Trend, der bei der Temperaturdifferenz ${ }_{m}{ }^{t}$ min $z$ wischen der Hohen Warte und Krems an der Donau im Zeitraum 1953-1972 für die Station 3807 gefunden wurde, ist kein „Klimatrend“, sondern hängt wahrscheinlich mit der Alterung eines Sensors oder der Wetterhütte zusammen. Man hat zwei Möglichkeiten diesen zu behandeln. Entweder man vernachlässigt diesen Trend und berechnet die mittlere Differenz aus diesem Zeitraum. Wenn man aber eine Alterung unterstellt, ist es richtiger, diesen Trend herauszurechnen. Im vorliegenden Fall wurde so vorgegangen, dass angenommen wurde, dass der Mittelwert der ersten drei Jahre noch keine „alterungsbedingten Messfehler“ aufweist. Basierend auf dieser Annahme wurde eine Trendbereinigung durchgeführt und die Berechnung der Korrekturfaktoren durchgeführt.

2. Bestimmung der Korrekturfaktoren für ${ }_{\mathrm{m}} \mathrm{t}_{\min }$ und ${ }_{\mathrm{m}} \mathrm{t}_{\max }$, d.h. Ermittlung des Wertes, der von der Messreihe der Hohen Warte als „quasikonstante Differenz“ in Abzug gebracht wird, um auf die Werte von Krems an der Donau zu schließen.

Basierend auf den homogenisierten Daten wurden die weiteren Berechnungen der Temperatur- und Wärmesummentrends für Krems an der Donau vorgenommen.

\subsection{Temperatur, Wärmesummenindex und Eintritt der Rebblüte}

Als Analyseperiode wurde der Zeitraum ab 1971-2006 bzw. 2007 gewählt. Dies soll die Vergleichbarkeit mit anderen Studien sicherstellen, die 1971 als Startjahr verwendet haben (vgl. z.B. DuchêNe \& SCHNeIDER 2005), und 40 Jahre entsprechen in etwa der Standzeit einer Rebe und sind somit weinbaulich relevant (vgl. Tab. 4).

Die Analyse der Daten für Krems an der Donau im Zeitraum 1971-2006 hat für alle analysierten Perioden einen signifikanten Anstieg der Temperatur ergeben. Der größte Temperaturanstieg hat am Beginn der Vegetationsperiode (April - Juni) stattgefunden, was direkte Auswirkungen auf den Beginn der phänologischen Phasen hat. So ist es im Zeitraum 1971-2007 zu einer Verfrühung der Blüte um elf Tage gekommen (vgl. Abb. 4). Das heißt einerseits, dass die Rebe mehr Zeit für die physiologische Entwicklung hat, andererseits aber auch zunehmend mit extremen Bedingungen ${ }^{5}$ kämpfen muss. Außerdem bedeutet eine Verfrühung der phänologischen Phasen, dass die Reifung der Trauben unter zunehmend heißen Bedingungen erfolgt und der für Spitzenweine benötigte Wechsel zwischen warmen Tagen und kühlen Nächten nur mehr bedingt zur Verfügung steht.

\footnotetext{
5) Bei hohen Temperaturen und geringer Luftfeuchtigkeit schließt die Rebe die Blattporen und stellt die Assimilation ein (vgl. Gladstones 1992, S. 29).
} 


\begin{tabular}{|l|c|c|c|c|c|c|}
\hline & \multicolumn{2}{|c|}{ Jän - Dez } & \multicolumn{2}{c|}{ Apr - Jun } & \multicolumn{2}{c|}{ Apr - Okt } \\
\hline Parameter & tmin & tmax & tmin & tmax & tmin & tmax \\
\hline Anstieg in ${ }^{\circ} \mathbf{C}$ pro Dekade & $+0,25$ & $+0,42$ & $+0,43$ & $+0,76$ & $+0,38$ & $+0,60$ \\
\hline Anstieg in $^{\circ} \mathbf{C}$ von 1971-2006 & $+0,92$ & $+1,55$ & $+1,61$ & $+2,80$ & $+1,31$ & $+2,20$ \\
\hline P-Wert $^{())}$ & 0,006 & 0,001 & $<0,001$ & $<0,001$ & $<0,001$ & $<0,001$ \\
\hline
\end{tabular}

Tab. 4: Anstieg der Temperatur $\mathrm{t}_{\min }$ und $\mathrm{t}_{\max }$ für ausgewählte Perioden im Zeitraum 1971-2006

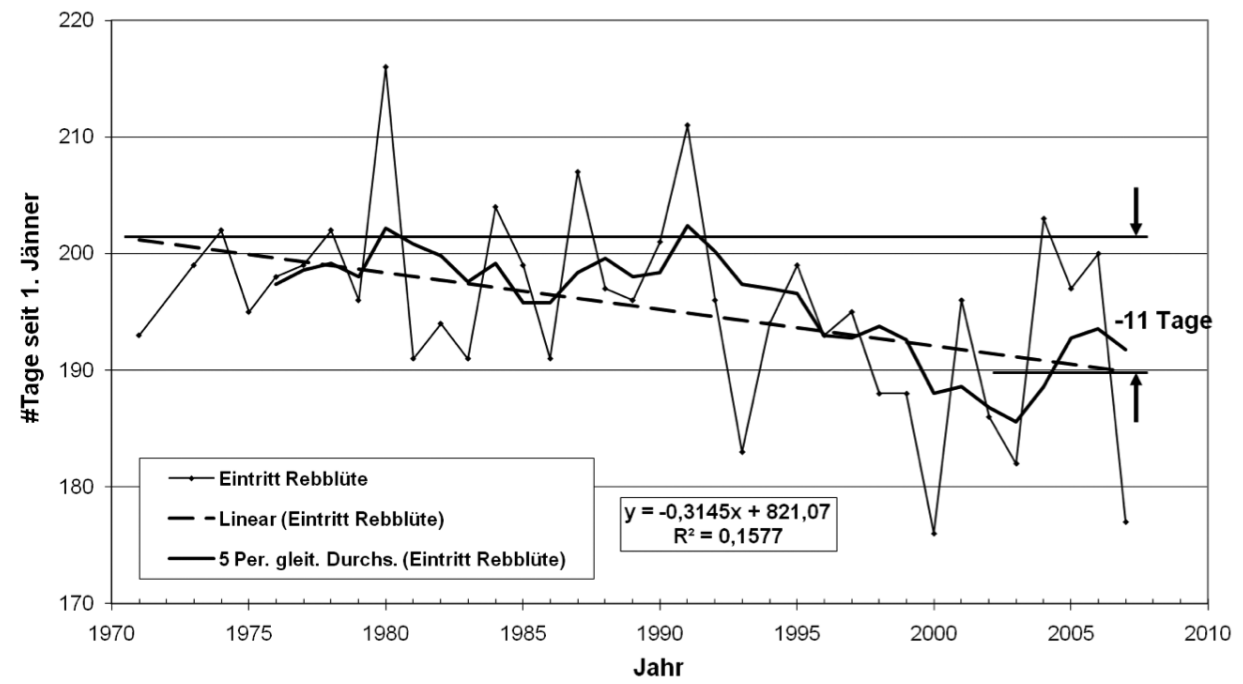

Abb. 4: Verfrühung der Rebblüte um elf Tage im Zeitraum 1971-2007 in Krems an der Donau

Die Wachau ist, wie andere Spitzenweinbaugebiete der Welt, eine Region mit einem „kühlen Randklima“ (vgl. Jones 2003, S. 3), in dem die angebauten Rebsorten gerade zur Reife gebracht werden können, zumindest unter den Bedingungen der Vergangenheit. Dies kommt klar zum Ausdruck, wenn man die Entwicklung des Wärmesummenindex WI nach Gladstones betrachtet (vgl. Abb. 5). Nach Gladstones (1992, S. 67) benötigt Riesling in etwa eine Wärmesumme von 1.200, Grüner Veltliner

\footnotetext{
6) Der P-Wert gibt die Wahrscheinlichkeit eines Fehlers erster Art an, also die Wahrscheinlichkeit, eine gültige Nullhypothese zu verwerfen, wenn man die Nullhypothese aufgrund der beobachteten Daten ablehnt. Je kleiner der P-Wert, desto eher sollte die Nullhypothese verworfen werden. Für alle Auswertungen wurde als Null-Hypothese kein signifikanter Zusammenhang zwischen der abhängigen Variable und der unabhängigen Variable unterstellt. Ein niedriger P-Wert gibt somit an, dass die Nullhypothese verworfen werden muss, was bedeutet, dass ein signifikanter Zusammenhang besteht. Das Signifikanzniveau wird durch die Höhe des P-Werts angegeben. Zum Beispie bedeutet ein P-Wert von 0,006 ein Signifikanzniveau von $99,4 \%$, d.h. es gibt eine sehr hohe Wahrscheinlichkeit, dass ein signifikanter Zusammenhang zwischen den untersuchten Variablen besteht.
} 


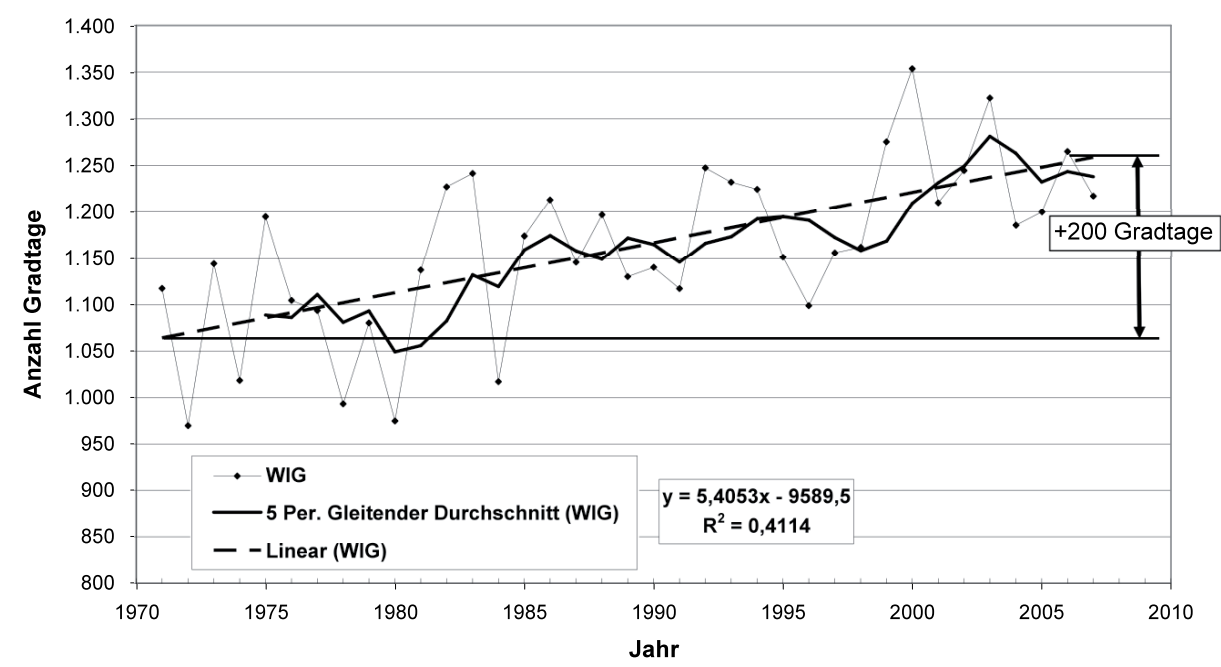

Abb. 5: Veränderung des Wärmesummenindex WI ${ }_{G}$ nach GladstonEs von 1971-2007 (April - Oktober) in Krems an der Donau

1.150. Der $\mathrm{WI}_{\mathrm{G}}$ liegt im Durchschnitt der Jahre 1998-2007 bereits bei 1.244. Je nach Lage eines Weinberges müssen die errechneten Werte noch korrigiert, d.h. an die lagenspezifischen Gegebenheiten (Ausrichtung, Geländeform, Gewässernähe, etc.) angepasst werden. Die Korrekturfaktoren für die Anpassung an die Lage wurden auf den höchsten Wert eingestellt. Das heißt, es wurden die Werte für die Spitzenlagen der Wachau - mit hoher Exposition, Südausrichtung und Steinterrassen - berechnet. Der $\mathrm{WI}_{\mathrm{G}}$ muss für die Periode 1998-2007 noch zusätzlich um +46 (Berechnung entsprechend Gladstones 1992, S. 69ff.) erhöht werden, da in der allgemeinen Berechnung die Lageneffekte noch nicht berücksichtigt sind. Der $\mathrm{WI}_{\mathrm{G}}$ liegt somit für die nach Süden ausgerichteten Spitzenlagen der Wachau bei 1.290. Das Modell deutet darauf hin, dass es für die Spitzensorten der Wachau, den Riesling und den Grünen Veltliner, langsam zu warm werden könnte.

\subsection{Zukünftige Temperatur}

Die Daten für die Darstellung der zukünftigen Temperatur sowie der erwarteten Temperaturtrends stammen aus dem Projekt reclip:more (reclip:more 2007b). reclip:more wurde initiiert, um eine Grundlage für Klimaschutz und Klimafolgenforschung in Österreich zu schaffen. Sollen Aussagen über regionale Klimaänderungen und ihre Auswirkungen getroffen werden, müssen die Ergebnisse einer globalen Klimaberechnung auf lokale Verhältnisse übertragen werden. Dies geschieht dadurch, dass regionale Klimamodelle RCM (Regional Climate Model) mit hoher Detailinformation einer Region in globale Modelle eingebettet werden; diese Einbettung wird „Nesting“ genannt (vgl. ЈАСОВ 2006). 
Bei der Interpretation der Ergebnisse von Klimamodellen ${ }^{7)}$ muss berücksichtigt werden, dass es sich dabei um keine Vorhersagen (Prognosen) über den zukünftigen Verlauf lokaler und globaler Klimata handelt, sondern um mögliche Szenarien, welche sich aufgrund der Vorannahmen über die zukünftige Entwicklung verschiedener Parameter wie zum Beispiel Emissionen oder Landnutzung ergeben. Eine der zentralen Fragen in der Klimamodellierung ist, wie sich die Emissionen in Zukunft entwickeln werden, da diese mit dem anthropogen verursachten Treibhauseffekt unmittelbar in Verbindung stehen. Die zukünftigen Emissionen sind von den sozialen, ökonomischen und politischen Entwicklungen abhängig und können grundsätzlich nicht vorausgesagt werden. Die Wissenschafter behelfen sich damit, dass sie mittels einer Bandbreite von Annahmen über die zukünftige Entwicklung der Menschheit eine Vielzahl von Emissionsszenarien ableiten, die als Grundlage für die Klimamodellierung herangezogen werden. Die in den Berichten des Intergovernmental Panel on Climate Change (IPCC) angeführten SRES-Szenarien (Special Report on Emission Scenarios) sind plausible Annahmen über die möglichen Entwicklungen im 21. Jahrhundert in den Bereichen Bevölkerungswachstum, ökonomische und soziale Entwicklung, technologische Veränderungen, Ressourcen-Verbrauch und Umweltmanagement.

Bei der Interpretation der Ergebnisse des reclip:more-Projektes ist zu beachten, dass als Antrieb für die Regionalisierung nur ein Emissionsszenario (IS92a) und nur ein General Circulation Model (GCM) herangezogen wurden. Die Ergebnisse liegen nur als „Punktresultat“ und nicht als „Ergebnisraum“ vor. Dies wurde im Bericht auch explizit angeführt, und die Verwendung mehrerer globaler Klimaszenarien sowie alternativer GCM-Läufe wurden als vordringliche Folgearbeit definiert. Es handelt sich um ein mögliches Bild der zukünftigen Verhältnisse, das leider (noch) nicht mit anderen „Bildern der Zukunft“ verglichen werden kann. Die Resultate aus reclip:more sind plausibel, speziell wenn man die Entwicklung der Temperatur betrachtet. Der Vergleich verschiedener Modellprojektionen - unter Verwendung unterschiedlicher GCMs und Emissionsszenarien - zeigt, dass die über ein Jahrzehnt gemittelte Erwärmung bis 2030 auf allen bewohnten Kontinenten nicht wesentlich von der Wahl des Emissionsszenarios abhängig ist und alle Modelle einen weiteren Anstieg der Temperatur projizieren (vgl. IPCC 2007, S. 13).

Die regionalen Ergebnisse aus den RCMs ergeben für Krems an der Donau einen Anstieg der Durchschnittstemperatur in den 2040ern um ca. $+2,3{ }^{\circ} \mathrm{C}$ gegenüber den 1980ern. Die am unmittelbarsten ableitbare Konsequenz daraus ist der Anstieg der

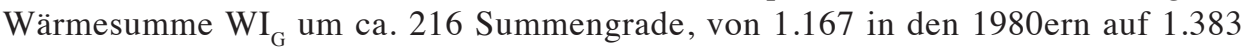
in den 2040ern. Die Wärmesumme berücksichtigt noch keine Korrekturfaktoren (der errechnete Wert ergibt ca. +27 Summengrade), womit in den 2040ern eine Wärmesumme $\mathrm{WI}_{\mathrm{G}}$ von 1.410 erreicht wird.

\footnotetext{
7) Bei Klimasimulationen erfolgt zuerst eine Kontrollsimulation, die das heutige Klima widerspiegelt. Dabei wird das Modell mit den herrschenden Bedingungen verglichen und bei guter Übereinstimmung erfolgt danach ein Klimaänderungsexperiment, wie z.B. durch Vorgabe eines Anstiegs der Kohlendioxidkonzentration. Um die Klimaänderung zu bestimmen, werden dann die ursprüngliche und die Klimaänderungssimulation zueinander in Beziehung gesetzt. Dieses Verfahren wird mehrfach durchgeführt. Aufgrund der wiederholten Simulationen mit veränderten Anfangsparametern erhält man ein statistisches Ensemble (= Ergebnisraum der möglichen klimatischen Zustände in der Zukunft) und kann dadurch auch eine Aussage über die Unsicherheit der Modellergebnisse treffen (vgl. auch SVGUA 2003, S. 25ff.).
} 


\subsection{Toleranzbereich des Systems}

Die meisten Systeme können Bedingungen, die vom Mittelwert abweichen, bis zu einem gewissen Grad verarbeiten, ohne Schaden zu nehmen. Dieser Bereich ist der sogenannte Toleranzbereich. Er markiert die obere und untere Trennungslinie, ab der ein Ereignis nicht mehr Ressource, sondern Gefahr für ein System darstellt. Wenn durch die Klimaänderung die Temperaturen steigen, sollte auch eine Aussage getroffen werden, welche Toleranzgrenzen ein System aufweist, d.h. bis zu welchem Grad das System in der Lage ist, von den Mittelwerten abweichende Bedingungen zu tolerieren.

In der „Welt des Weines“ wird die Identität eines Weinbaugebietes maßgeblich durch die Produktqualität und -integrität im Sinne von Konsistenz der angebauten Rebsorten, Weinstil (Aroma, Geschmack, Struktur der Weine) und Wein-Komposition bestimmt (vgl. HAYEs 2007). Durch die Temperaturänderung kommt es zu Veränderungen in der Produktqualität und -integrität, was dazu führen wird, dass sich auch die Identität des Weinbaugebietes, welche über viele Jahrzehnte geschaffen wurde, verändern wird. Ein Wein, der unter warmen oder heißen Bedingungen entsteht, hat gänzlich andere Charakteristika als ein Wein, der unter kühlen Temperaturbedingungen produziert wird.

Gladstones hat in seiner Arbeit auch den Zusammenhang zwischen den Temperaturbedingungen und den daraus resultierenden Weinstilen untersucht. Er hat seine Analysen auf Basis der Anzahl der Sonnenstunden und der Temperatur durchgeführt und kommt zu folgendem Ergebnis (vgl. auch Abb. 6): bis zu einer Wärmesumme WI von 1.450 ist die Temperatur der bestimmende Faktor, welcher Weinstil produziert werden kann. Die Wärmesumme bestimmt, ob eine spezifische Rebsorte überhaupt zur Reife gebracht werden kann, da in den meisten „,kühlen Weinbauklimata“ die Minimalanforderungen an die Anzahl der Sonnenstunden ${ }^{8)}$ immer erreicht werden. Bis zu einer Wärmesumme von 1.450 sind die potenziellen Weinstile auf „Tafelweine“"9) beschränkt. Darüber hinaus bestimmt das Verhältnis der Wärmesumme zur Anzahl der Sonnenstunden den potenziellen Weinstil, der unter diesen bestimmten klimatischen Verhältnissen hervorgebracht werden kann (für eine detaillierte Darstellung vgl. Gladstones 1992, S. 20ff.).

In Abbildung 6 sind viele der wichtigsten Weinbaugebiete der Welt (jeder Punkt entspricht einem Weinbaugebiet) dargestellt. Die Werte für Krems an der Donau und das Gebiet von Bordeaux wurden für die Periode 1971-2000 neu berechnet. Die Ordinate entspricht der Anzahl von Sonnenstunden und die Abszisse der Wärmesumme. Der Bereich oberhalb der Linie AA gibt die minimale Anzahl von Sonnenstunden in Abhängigkeit von der Wärmesumme an, die benötigt wird, um einen Tafelwein über die 7-Monats-Periode zu produzieren. Die Linie BB gibt den Übergang zu „full-bodied“ (vollmundigen) Weinstilen an.

8) $1.250 \mathrm{~h}$ im Zeitraum April - Oktober (vgl. BeCKer 1977).

9) Tafelwein im Sinne eines gut balancierten „Bordeaux-Stils“, d.h. nicht übermäßig kräftig, mit Finesse durch das balancierte Verhältnis der verschiedenen Weinkomponenten (im Speziellen Frucht, Gerbstoffe, Säure, Alkohol). 


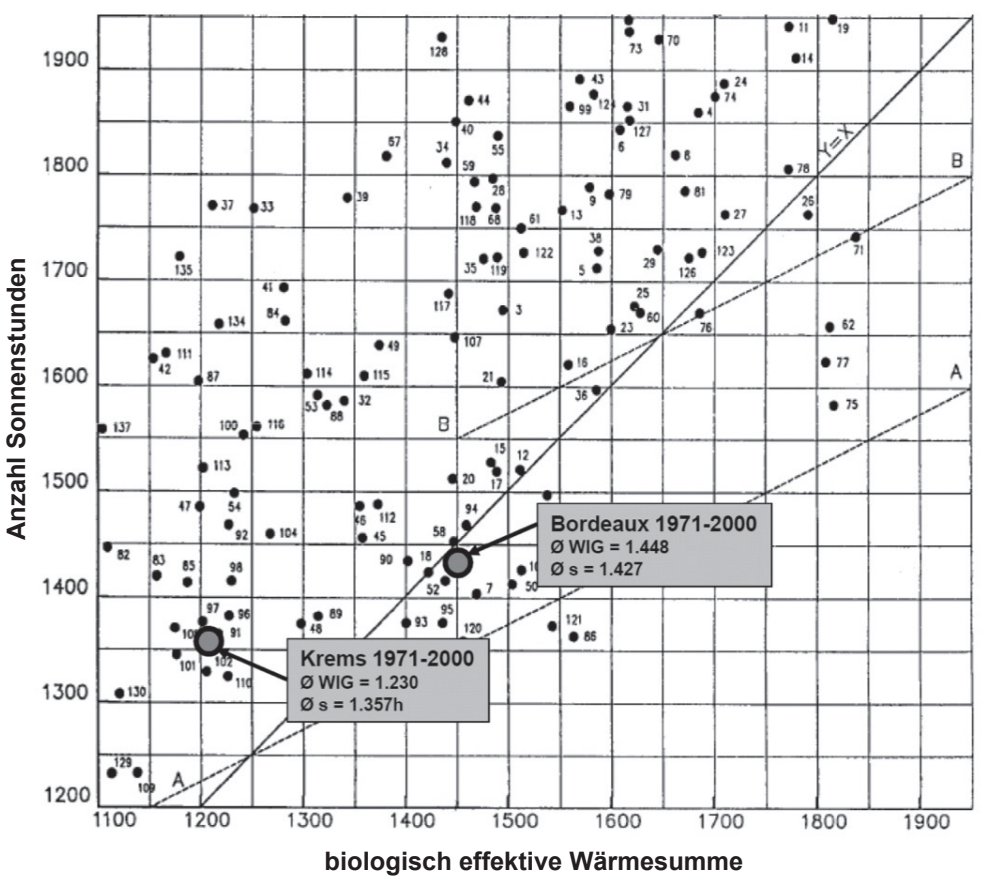

Quelle: Gladstones (1992, S. 24); ergänzt und aktualisiert durch den Verfasser

Abb. 6: Anzahl Sonnenstunden versus Anzahl Gradtage für den Zeitraum April - Oktober

Zur Herleitung der möglichen Auswirkungen der Temperaturänderung auf die Wachau wird geprüft, welches Weinbaugebiet im vergangenen langjährigen Durchschnitt der zukünftigen, durch die Veränderung der Temperatur herbeigeführten Position entspricht. Man kann so durch Analogieschluss eine Einschätzung der zukünftigen Situation vornehmen. Dazu wurden aus dem zur Verfügung stehenden Datenmaterial und dem reclip:more-Projekt die in Tabelle 5 angeführten Temperaturparameter für die Vergangenheit und die Zukunft für Krems an der Donau berechnet und in den Raster der Abbildung 7 übertragen.

\begin{tabular}{|l|c|c|c|}
\hline & $\mathbf{1 9 8 1 - 1 9 9 0}$ & $\mathbf{1 9 9 8 - 2 0 0 7}$ & $\mathbf{2 0 4 0 e r}$ \\
\hline Anzahl Sonnenstunden (s) & 1.313 & 1.404 & $1.587^{10)}$ \\
\hline Anzahl Gradtage (WIG) & 1.230 & 1.290 & 1.410 \\
\hline
\end{tabular}

Tab. 5: Anzahl Sonnenstunden und Gradtage vergangener und zukünftiger Vegetationsperioden (April - Oktober) für Krems an der Donau

\footnotetext{
${ }^{10)}$ Es wurde unterstellt, dass sich die Zunahme der Anzahl der Sonnenstunden in Abhängigkeit der Wärmegradtage linear mit gleicher Steigung fortsetzt. Aus dieser Vorgangsweise - zur besseren Darstellung der möglichen Verschiebung der klimatischen Verhältnisse - darf aber nicht geschlossen werden, dass eine Zunahme der Temperatur automatisch auch mit einer Zunahme der Anzahl der Sonnenstunden verbunden sein muss.
} 


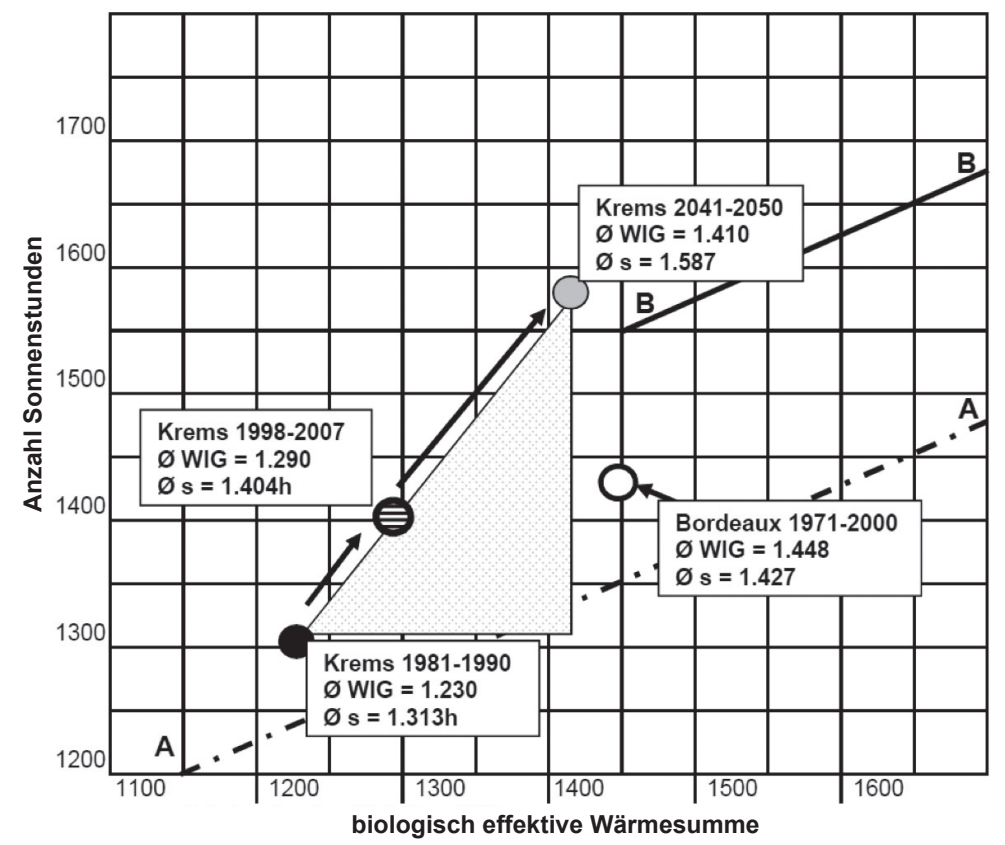

Abb. 7: Mögliche zukünftige klimatische Verhältnisse für die Vegetationsperiode (April - Oktober) in der Wachau (die Linien AA und BB entsprechen jenen in Abbildung 6)

In Abbildung 7 ist ersichtlich, wie eine mögliche Entwicklung der Temperaturverhältnisse in der Wachau aussehen könnte. Das graue Dreieck stellt einen „Ergebnisraum“ dar, der die möglichen (auf linearer Fortschreibung der Anzahl der Sonnenstunden ermittelten) Verhältnisse der Wachau in der Zukunft darstellt. Wie man sieht, ist zum Beispiel die Zunahme der Wärmesumme für die Periode 1998-2007 auch mit einer höheren Anzahl von Sonnenstunden einhergegangen. Dies wird auch durch die Analyse der Daten der Hohen Warte bestätigt. Im Zeitraum von 1971-2007 ist die Anzahl Sonnenstunden um $383 \mathrm{~h}$ gestiegen (P-Wert <0,001).

Zur Herleitung der Analogie - und zur Diskussion des Toleranzbereichs des Systems - wurde ein Punkt in der Grafik gesucht, der in etwa der zukünftigen Wärmesumme und Anzahl der Sonnenstunden von Krems an der Donau entspricht. Der so gefundene Punkt entspricht dem wohl berühmtesten Weinbaugebiet der Welt, dem Gebiet von Bordeaux. Das Gebiet von Bordeaux weist im 30-jährigen Durchschnitt (1971-2000) eine Wärmesumme von 1.448 sowie 1.427 Sonnenstunden auf und entspricht damit recht genau der für die Wachau ermittelten zukünftigen Situation in den 2040ern. Bei den Rebsorten dominieren im Gebiet von Bordeaux Cabernet Sauvignon, Merlot und Cabernet Franc, alles rote Rebsorten, die eine Wärmesumme von 1.250 (Merlot, Cabernet Franc) bzw. 1.300 (Cabernet Sauvignon) benötigen. Auf den ersten Blick mag es erscheinen, dass die Rebsorten im Gebiet von Bordeaux nicht sonderlich gut an die vorhandenen Temperaturverhältnisse angepasst sind, übersteigt doch die vorhandene 
Wärmesumme von 1.448 die benötigte Wärmesumme von 1.250 (Merlot und Cabernet Franc) bzw. 1.300 (Cabernet Sauvignon) um 198 bzw. 148 Wärmesummengrade. Die Weinlese erfolgt im Gebiet von Bordeaux traditionell im Monat September, beginnt aber häufig schon im August und kann sich manchmal bis Oktober hinziehen. Das heißt, dass nicht die gesamte zur Verfügung stehende Vegetationsperiode genützt wird. In der Periode von April bis Ende September werden ca. 1.320 Wärmesummengrade erreicht, was einer perfekten Anpassung der Rebsorten an die bestehenden Temperaturverhältnisse entspricht.

\section{Ausblick}

Was kann daraus für die Wachau geschlossen werden? Einerseits, dass es zu warm werden könnte für die Leitsorten Grüner Veltliner und Riesling, und dass ein Rebsortenwechsel notwendig werden kann. Die Top-Weinbaugebiete der Welt zeichnen sich dadurch aus, dass die angebauten Rebsorten sehr genau an die Temperaturverhältnisse eines Weinbaugebietes angepasst sind (vgl. JonEs 2003). Wenn die durchschnittlichen Verhältnisse des Gebiets von Bordeaux (für die Wärmesumme und die Anzahl der Sonnenstunden) im Zeitraum 1971-2000 denen der Wachau in den 2040ern entsprechen, dann kann argumentiert werden, dass das System mit den derzeit angebauten Rebsorten den Toleranzbereich verlässt oder, anders ausgedrückt, aus dem Gleichgewicht oder Optimum gerät. Ein solcher Anstieg der Wärmesumme würde früh zu hohen Zuckerwerten und damit zur Notwendigkeit einer früheren Ernte führen. Dann haben die Trauben jedoch nicht den Wechsel von warmen und kalten Tagen des Herbstes, sie enthalten nicht genug Säure und Aromastoffe, der Wein wird flach. Ein weiteres Problem, das auf die Winzer zukommt, ist, dass die Trauben im warmen Zustand in den Keller kommen und eine andere Gärführung erfordern als es bei kühlem Traubenmaterial der Fall ist. Bei einer solchen Entwicklung wird es aller Wahrscheinlichkeit nach zu einer Verschiebung der Spitzenlagen kommen; heute (kühle) „Ungunstlagen“ wären die Gewinner einer wärmeren Zukunft. In den Spitzenlagen wird es wahrscheinlich zu Sortenänderungen kommen - hin zu Rebsorten, die besser an die wärmeren Bedingungen angepasst sind.

\section{Literaturverzeichnis}

BECKER N. (1977), Selection of vineyard sites in cool climates. Allbury.

Boulton R.B., Singelton V.L., Bisson L.F., Kunkee R.E. (1998), Principles and practices of winemaking. Gaithersburg, Md., Chapman \& Hall.

DuchêNe E., SCHNEIDER C. (2005), Grapevine and climatic changes: a glance at the situation in Alsace. In: Agronomic Sustainable Development, 25, S. 93-99. 
Gladstones J. (1992), Viticulture and Environment. Adelaide, Winetitles.

HAYES P. (2007), Global Climate Change-Implications for Viticulture and Oenology. Intervitis Interfructa $2007,8^{\text {th }}$ International Symposium, Stuttgart.

Intergovernmental Panel on Climate Change (IPCC) (Hrsg.) (2007), Klimaänderung 2007 Wissenschaftliche Grundlagen: Zusammenfassungen für politische Entscheidungsträger. United Kingdom - New York, Cambridge Univ. Press.

JАсов D. (2006), Klimawandel: Globale und regionale Klimamodelle. - http://www.umwelt dachverband.at/NG_100906/NG_100906pdfs/Jacob.pdf (25.09.2008)

Jones G. (2003), Climate and Terroir: Impacts of Climate Variability and Change on Wine. In: Geological Society of America (Hrsg.), Terroir, geology and wine: a tribute to Simon J. Haynes, S. 14. Seattle, The Geological Society of American Annual Meeting.

Jones G. (2006), Climate change and wine: Observations, impacts and future implications. In: Wine Industry Journal, 21, 4, S. 21-26.

Koch E., Scheifinger H. (2004), Phänologie ein Bio-Indikator für den Klimawandel. Irding.

Olberg M., Stellmacher R., Kondrat‘ev K.J. (1991), Klimadaten. In: Bernhardt K., Hupfer P. (Hrsg.), Das Klimasystem der Erde - Diagnose und Modellierung, Schwankungen und Wirkungen, Berlin, Akademie Verlag.

Österreich Wein Marketing GmbH (ÖWM) (Hrsg.) (2006), Dokumentation Österreichischer Wein. Wien.

Priewe J. (1998), Wein. München, Zabert Sandmann.

reclip:more (2007a), Research for Climate Protection. - http://systemsresearch.arcs.ac.at/SE/ projects/reclip/ (13.01.2008)

reclip:more (2007b), Klimazukunft Österreich - Medieninformaton zum Projektabschluss. http://tbd (14.03.2008)

SachVerständigenkreis „Globale Umweltaspekte” (SVGUA) (Hrsg.) (2003), Herausforderung Klimawandel. Berlin, BM f. Bildung u. Forschung.

Scheifinger H., Böhm R., Auer I. (2003), Räumliche Dekorrelation und Homogenisierbarkeit von Klimazeitreihen. In: Terra Nostra, 6, S. 375-379.

Zentralanstalt für Meteorologie und Geodynamik (ZAMG) (Hrsg.) (2007), Klimadaten Österreich - Krems. Wien.

Zentralanstalt für Meteorologie und Geodynamik (ZAMG) (Hrsg.) (2008a), Klimadaten Österreich - Wien. Wien.

Zentralanstalt für Meteorologie und Geodynamik (ZAMG) (Hrsg.) (2008b), StartClim1. http://www.zamg.ac.at/forschung/klimatologie/klimawandel/startclim1/?ts $=1224773401$ $(23.10 .2008)$ 
